Ann. Génét. Sél. anim., I973, 5 (2), I89-209.

\title{
RECHERCHE DES CONDITIONS DE RENTABILITÉ D'UN SCHÉMA DE SÉLECTION D'UNE SOUCHE DE BOVINS DESTINÉE AU CROISEMENT DE PREMIÈRE GÉNÉRATION POUR LA PRODUCTION DE VEAUX DE BOUCHERIE
}

\author{
J.-C. MOCQUOT et J.-L. FOULLEY \\ Station de Génétique quantitative et appliquée, \\ Centre national de Recherches zootechniques, I. N.R. A., \\ 78350 Jouy en Josas
}

\section{RÉSUMÉ}

A propos de ce schéma nous avons élaboré un mode de raisonnement génético-économique des opérations de sélection. Plus particulièrement nous avons envisagé la sélection des ascendants paternels de ces taureaux, donc la rentabilité à long terme, en comparant, au niveau d'un cycle élémentaire de sélection, 3 modes de choix des taureaux pères :

- choix sur performance individuelle en station (poids à 12 mois) ;

- choix sur index de descendance en ferme (poids à 3 mois) ;

- choix en 2 étapes par troncatures successives sur le poids à 12 mois en station puis sur un index combiné des 2 critères précédents.

Nous avons dans chaque cas tenu compte de la répartition des recettes dans le temps par le biais de l'actualisation et recherché les conditions d'obtention du bénéfice actualisé maximum puis nous avons analysé la sensibilité des valeurs obtenues aux variations d'un certain nombre de paramètres économiques ou génétiques. Les résultats conduisent aux conclusions suivantes :

Io la sélection des ascendants mâles justifie à elle seule, et dans tous les cas, les investissements réalisés ;

$2^{\circ} 1^{\prime}$ introduction de contraintes économiques dans l'étude génétique des schémas de sélection permet de comparer les méthodes proposées de façon plus objective et de déterminer pour chacune d'elles des optimums plus réalistes;

$3^{\circ}$ parmi les méthodes étudiées, la sélection en deux étapes nous semble la meilleure. D'abord parce qu'elle procure les bénéfices les plus importants pour un coût donné, ensuite parce qu'au voisinage de l'optimum la réponse est moins sensible à une variation éventuelle des paramètres mal connus ou fixés a priori;

$4^{\circ}$ enfin on montre qu'une sélection directe des taureaux utilisés en croisement permet de mieux rentabiliser les frais déjà engagés pour la sélection sur ascendance. 


\section{I. - INTRODUCTION}

A cause du développement de l'insémination artificielle, le choix des reproducteurs mâles, pour l'espèce bovine tout au moins, est de plus en plus sous la responsabilité d'un petit nombre d'organisations d'éleveurs. Cette tendance s'est affirmée encore plus depuis que la conservation des doses de sperme à basse température permet un stockage de longue durée et des échanges commerciaux importants entre régions et même entre pays. En contrepartie des avantages techniques et économiques que peut entraîner cette concentration des moyens et des pouvoirs de décision, les répercussions d'une politique de sélection inadaptée deviennent considérables. Admissibles dans le cas d'un éleveur isolé, parce qu'il agit en entrepreneur libre et donc responsable, les erreurs graves ne le sont plus dans le cas de centres de production de semence qui engagent, en fait, le devenir de toute une collectivité. C'est la justification des réglementations que tous les pays ont adopté à ce sujet, sous des formes diverses, par exemple pour l'estimation de la valeur génétique des reproducteurs.

Étant donné l'importance des capitaux mis en jeu et le délai de diffusion du progrès génétique dans la masse des élevages, qui rendent difficiles des modifications ou des ajustements de programme fréquents, il est apparu indispensable d'étudier, sur des bases objectives et a priori, l'ensemble des opérations réalisées par une unité de sélection. Se fixant comme critère le progrès génétique, RoBERTSON (I957) a montré qu'on pouvait calculer des valeurs pour les principaux paramètres (intensité de sélection et effectif des lots de descendantes) qui rendaient ce progrès maximum. Le but visé était, cependant, critiquable le progrès génétique étant le moyen et non le but de la sélection. Celle-ci doit permettre d'utiliser au mieux les ressources techniques et financières disponibles à un instant donné (en particulier nombre d'animaux contrôlés et coût du contrôle) pour un objectif économique, puisqu'il s'agit de rendre maximum la différence entre les gains escomptés chez les éleveurs et les frais réalisés par le centre de production de semence. A notre connaissance la première étude originale sur ce sujet a été publiée, il y a dix ans (PouTous et VISSAC, I962). Par la suite ce type de recherches s'est développé surtout dans le cas de sélection des races et souches à aptitudes mixtes pour améliorer indépendamment ou simultanément la production laitière et la croissance (SOLLER, BAR ANAN et PASTERnAK, I966 ; LiNdHE, I968 ; Hinks, I970 $a$ et $b$; Mc ClinTock et CunNinGHAM, I97I ; HILL, I97I). Toutes utilisent la méthode d'actualisation des coûts et des recettes. Cette technique permet de comparer la rentabilité d'investissements, sur une période donnée, en intégrant, par le biais d'un taux d'intérêt, le facteur temps dans les comparaisons. Parmi toutes les options possibles, la méthode d'actualisation s'avère donc bien adaptée pour juger de l'efficacité d'un plan d'amélioration génétique à moyen et long terme (HILL, I97I).

Abordant souvent le même thème mais établies pour des structures de sélection et des conditions économiques propres à chaque pays, les conclusions de ces travaux sont difficilement transposables ailleurs. Pour rationaliser les choix budgétaires réalisés par les responsables de 1'économie, des études analogues apparaissent, donc, nécessaires à l'échelon national ou régional. C'est ce que nous avons essayé de 
réaliser dans le cas de la production de veaux de boucherie en croisement industriel, pour répondre à la demande d'un centre de production existant, compte tenu des contraintes réelles auxquelles il est soumis.

\section{II. - REMARQUES PRÉLIMINAIRES}

Schématiquement on peut considérer que la viande bovine produite en France est obtenue à partir de 3 populations : femelles traites qui se reproduisent en race pure; femelles non traites de races laitières, mixtes ou rustiques conduites pour une production dominante de veaux de boucherie par croisement de première génération avec des taureaux de races à viande; enfin femelles de races pures de boucherie dont proviennent les mâles utilisés pour le croisement dans le cas précédent. Cette diversité d'orientations conduit à envisager des schémas de sélection différents pour chacune d'entre elles (VISSAC et al., I97I). Si certaines études réalisées à l'étranger (LINDHE, I968 ; Hinks, I970-I97I ; HiLl, I97I ; MC ClinTock et CunNINGHAM, I97I) apportent, dans une certaine mesure, des éléments de réponse aux problèmes soulevés par l'amélioration de la production de viande dans les troupeaux laitiers soumis à la traite, ceux relatifs à la production de viande en troupeaux de femelles allaitantes soumises au croisement n'ont pas encore été étudiés de ce point de vue économique et feront l'objet de notre recherche.

En pratique la sélection des taureaux de race à viande destinés au croisement industriel (centre et sud-ouest de la France) comporte trois stades qui ont été mis en place dans l'ordre chronologique suivant :

- choix sur descendance croisée contrôlée en ferme en fonction de la production dominante de la zone : veaux de boucherie élevés au pis (I960-65);

- choix sur propre performance contrôlée en station de sélection du sevrage à I3-I4 mois (I964-67) ;

- choix sur ascendance paternelle dans le cadre d'accouplements contractuels (r968-7I).

Ce processus chronologique de développement du schéma de sélection s'explique par le fait que les centres d'insémination situés dans des zones exclusives de croisement n'ont acquis que progressivement la maitrise complète du choix des reproducteurs de race pure, choix primitivement réalisé sur des animaux adultes à l'occasion des concours (FREBLING, GaIllaARD et VISSAC, I97I). Au terme de ce cheminement lent et à première vue illogique, les centres contrôlent l'ensemble du schéma, il apparaît donc opportun d'entreprendre l'étude économique de synthèse destinée à déterminer ses conditions optimales de réalisation compte tenu des installations nécessaires à la sélection, de la législation française (loi sur l'élevage, votée en I966) et des données technologiques relatives à l'utilisation de la semence.

\section{III. - CONDITIONS DE RÉALISATION DE L'ÉTUDE}

\section{A. - Considérations générales}

Nous considérerons une population de femelles inséminées comportant : une majorité de femelles support de croisement de première génération (fraction A) dont les produits mâles et femelles sont conduits sous la mère et commercialisés à l'âge de 3 mois en tant que veaux de 
boucherie. Cette première sous-population (A) sera donc renouvelée par apport extérieur de femelles de races rustiques, mixtes ou laitières, éliminées de la traite du fait de la sélection laitière. La seconde fraction (B) est constituée d'un noyau de femelles de race à viande se renouvelant en circuit fermé et dont les accouplements sont planifiés pour produire les mâles de race pure destinés à l'insémination, les mâles non retenus étant élevés en veaux de boucherie.

Nous avons vu que le choix des reproducteurs, parmi les mâles candidats à la sélection, peut être effectué sur différents critères (croissance, conformation...) et à différents stades
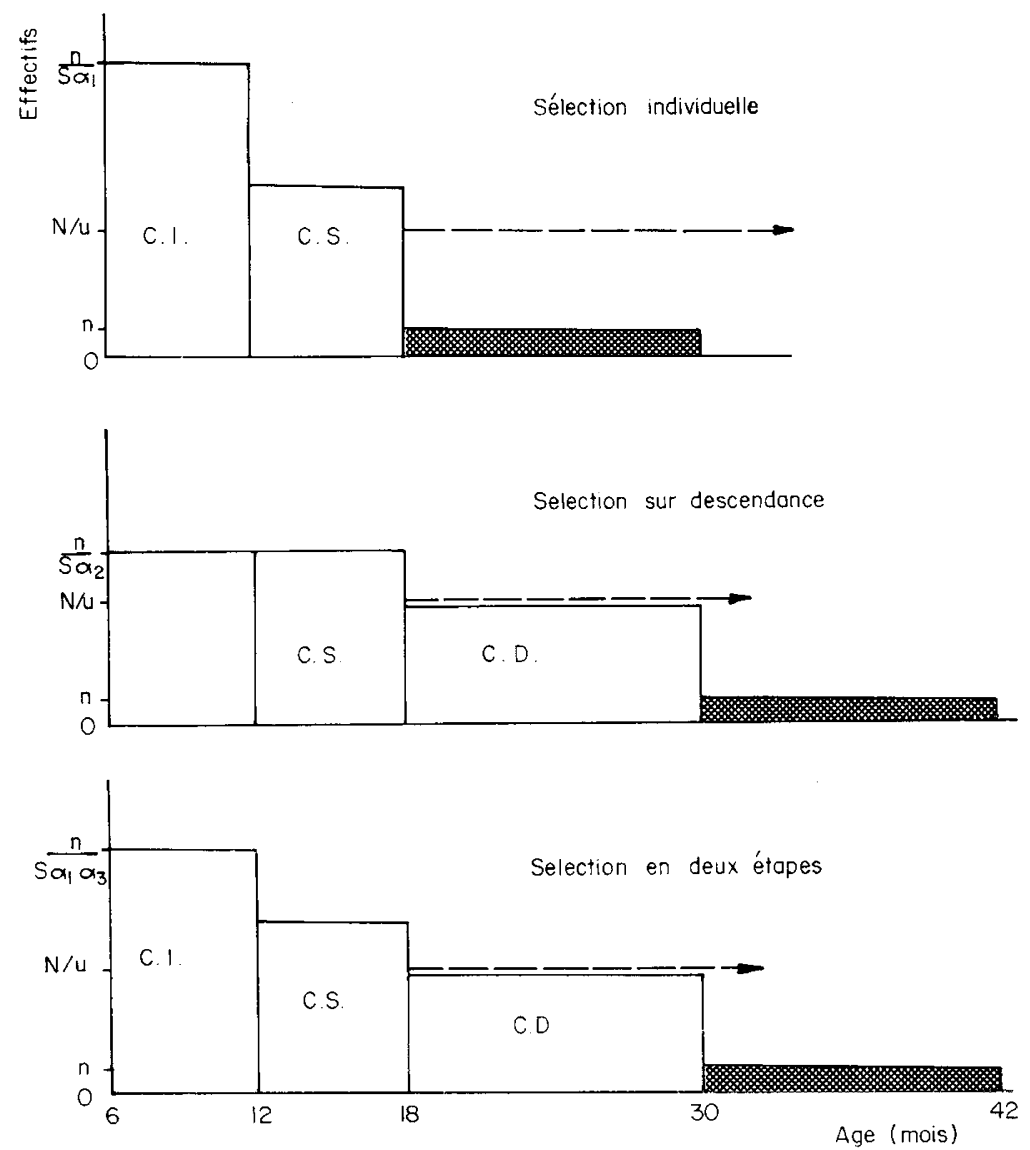

FIG. I. - Déroulement des différents cycles de sélection

CI contrôle individuel

CS contrôle de la production de sperme

$\mathrm{CD}$ contrôle de descendance

$\rightarrow$ utilisation en croisement

utilisation en race pure

(choix sur performance individuelle en ferme ou en station, choix sur descendance en ferme, etc.).

Nous avons comparé les trois schémas de sélection suivants, pour les taureaux utilisés en race pure et destinés à produire la génération suivante de jeunes mâles (fig. I) :

- choix sur performance individuelle en station de sélection (poids à I 2 mois) ;

- choix sur index de descendance en ferme (poids à 3 mois des descendants) ;

- choix en 2 étapes, un premier choix étant effectué sur le poids à I 2 mois en station ; le deuxième choix porte sur un index combiné du poids individuel à $\mathrm{r} 2$ mois et du poids à 3 mois des descendants. 
En ce qui concerne les taureaux utilisés pour le croisement nous avons, dans cette première étude, supposé qu'ils n'étaient en aucun cas choisis sur descendance, le tri se faisant à I 8 mois après élimination pour la production de sperme et, éventuellement, après contrôle individuel en station, mais sans sélection. Par ailleurs, nous avons admis, une durée $(u)$ d'utilisation pour le croisement industriel constante pour tous ces mâles retenus. Dans l'hypothèse d'une production de sperme constante par unité de temps pour chaque reproducteur, la valeur de $u$ fixe le nombre de taureaux nécessaires au centre. Quant aux maîtres d'œuvre de telles opérations nous distinguerons, comme le prévoit la législation française, le centre de mise en place de la semence qui pratique les inséminations et le centre de production responsable des opérations de sélection. Ce dernier a notamment la charge des accouplements contractuels réalisés sur la population $B$ de femelles ainsi que de la mise en contrôle d'aptitude, tant individuel que sur descendance, des veaux mâles issus de ces accouplements.

Du point de vue économique, l'étude des 3 options de sélection envisagées sera faite au niveau d'un cycle élémentaire de sélection visant à produire d'une part les taureaux utilisés en race pure et renouvellés annuellement d'autre part les taureaux destinés au croisement et utilisés dans les conditions définies précédemment. Nous considérerons, à ce propos, l'entité économique constituée par le centre de production et par ses adhérents, regroupés en général sous forme coopérative. Cette unité supporte des frais, engagés au niveau du centre de production, et afférents aux opérations de contrôle et de sélection. Ce sont en particulier les dépenses consenties pour l'amortissement des installations, l'achat, l'entretien et le contrôle des animaux, la récolte et la conservation du sperme, dépenses qui ne seraient pas nécessaires dans le cas d'utilisation de reproducteurs non sélectionnés. Elle réalise par ailleurs, du fait de la sélection et de l'utilisation de taureaux sélectionnés, des recettes mesurées par le revenu supplémentaire que procure aux éleveurs adhérents l'augmentation de production des veaux commercialisés. Ces recettes sont de deux types : les unes concernent le niveau génétique moyen des animaux utilisés en croisement une année donnée, les autres intéressant l'évolution de ce niveau du fait de la sélection. Ces dernières sont acquises de façon permanente et ne dépendent que du type et de l'intensité de sélection pratiqués pour le choix des taureaux utilisés sur le noyau $\mathrm{B}$ de femelles. Les premières, elles, sont imputables au mode de sélection et d'utilisation des taureaux issus du schéma et utilisés en croisement sur les femelles A. Nous nous sommes fixés comme but d'étudier la rentabilité comparée des méthodes de sélection proprement dites en ne considérant que les recettes supplémentaires imputables au progrès génétique annuel. Les coûts relatifs à l'utilisation des mâles en croisement ont ainsi été réduits à leur valeur minimale par l'hypothèse d'utilisation dès 18 mois des taureaux en croisement. Le second aspect de la rentabilité de telle ou telle méthode de sélection, c'est-à-dire la recherche d'un mode optimal d'utilisation en croisement, peut être traité de façon presque indépendante et sera discuté à la fin de l'étude.

\section{B. - Expression des gains dus à la sélection}

Dans le cadre restrictif qui a été défini, les gains dus à la sélection dépendent :

- de la supériorité génétique transmise à leurs descendants par les reproducteurs sélectionnés,

- du nombre d'animaux sur lesquels s'exprime la réponse à la sélection, donc du nombre de veaux de boucherie commercialisés,

- de l'intervalle de temps entre l'engagement des dépenses et l'encaissement des recettes.

a) Calcul du progrès génétique.

En considérant les quatre voies possibles de transmission des gènes d'une génération à la suivante le progrès génétique annuel s'établit de la façon suivante (RENDEL et RoBERTSON, I950) :

arec $j=\mathrm{I}$ du père au fils

$$
\Delta \mathrm{G}=\frac{\sum_{j=1}^{4} \Delta \mathrm{S}_{j}}{\sum_{j=1}^{4} \mathrm{~L}_{j}}
$$

$j=2$ du père à la fille

$j=3$ de la mère au fils

$j=4$ de la mère à la fille

$\Delta \mathrm{S}_{j}=$ supériorité génétique moyenne des parents sur la moyenne des candidats à la sélection pour la $j$ jème voie

$\mathrm{L}_{j}=$ intervalle de génération correspondant. 
L'application de cette formule au divers cas étudiés peut être faite moyennant un certain nombre d'hypothèses simplificatrices :

Les femelles du groupe $B$ sont sélectionnées, pour le caractère considéré, uniquement par la voie des mâles, celles du groupe A étant produites en dehors du schéma. Pour les mâles nous avons admis que les veaux entrant à 6 mois au centre de production constituent un échantillon non sélectionné des mâles produits par le noyau de femelles $B$. Cette hypothèse mériterait d'être levée au cours d'études ultérieures, puisque une sélection massale ou familiale précoce est en fait pratiquée. Nous avons par ailleurs considéré que les sélections successives au cours des différents cycles étaient effectuées par troncature sur la valeur de critères distribués normalement. Nous avons enfin admis que le nombre de descendants par taureau utilisé est indépendant de sa valeur génétique pour le caractère considéré. Ceci suppose en particulier l'indépendance génétique entre ce critère et les caractères de fertilité d'une part, une utilisation non sélective des taureaux conservés d'autre part.

Dès lors, dans les 3 cas étudiés ici, le progrès génétique résulte de la seule sélection pratiquée sur les mâles entrant en contrôle à 6 mois et visant à produire les $n$ taureaux réalisant les accouplements contractuels sur le noyau $\mathrm{B}$ de femelles de race à viande et $\mathrm{n}$ 'est transmis que selon 2 voies : Pères aux fils et pères aux filles avec la même efficacité pour chacune d'elles, cela entraîne :

et

$$
\begin{gathered}
\Delta \mathrm{S}_{1}=\Delta \mathrm{S}_{2} \\
\Delta \mathrm{S}_{3}=\Delta \mathrm{S}_{4}=\circ
\end{gathered}
$$

d'autre part nous avons admis que :

$$
\begin{aligned}
& \mathrm{L}_{1}=\mathrm{L}_{2} \\
& \mathrm{~L}_{3}=\mathrm{L}_{4}=5 \text { ans. }
\end{aligned}
$$

Si nous admettons que les progrès génétiques sont constants par unité de temps, permanents, et cumulatifs ceci suppose notamment : l'invariance des paramètres génétiques sous les effets de la sélection et de la consanguinité d'une part ; une sélection en phase continue et stable d'autre part. La première condition est illogique mais néanmoins acceptable sur une période de 20 ans qui correspond en moyenne à 4 ou 5 générations dans l'espèce bovine. La seconde condition n'est pas vérifiée pendant les premières années où l'évolution du niveau génétique est irrégulière, mais l'hypothèse de linéarité du progrès génétique cumulé en fonction du temps n'entraîne que de faibles biais (HILL, I97I).

Dès lors, pour chacun des systèmes de sélection étudiés nous pouvons exprimer le progrès génétique espéré en fonction des intensités de sélection pratiquées aux différents stades pour divers critères $\left(\mathrm{X}_{i}\right)$. Les expressions figurent au tableau $\mathbf{I}$ où $g$ désigne la valeur génétique du caractère à améliorer (poiđs à 3 mois) et $\sigma_{g}^{2}$ sa variance. $\mathrm{R}\left(g, \mathrm{X}_{i}\right)$ désigne la corrélation entre cette valeur $g$ et le caractère mesuré $\left(\mathrm{X}_{i}\right)$.

Pour la sélection en 2 stades le progrès génétique maximum est obtenu par troncature ${ }^{-}$au au $2^{\text {e }}$ stade sur l'index combiné de variance d'estimation minimale donc tel que :

$$
\begin{gathered}
\mathrm{X}_{3}=w_{1} \mathrm{X}_{1}+w_{2} \mathrm{X}_{2} \\
w_{1}=\frac{\mathrm{R}\left(g, \mathrm{X}_{1}\right)-\mathrm{R}\left(\mathrm{X}_{1}, \mathrm{X}_{2}\right) \cdot \mathrm{R}\left(g, \mathrm{X}_{2}\right)}{\mathrm{I}-\mathrm{R}^{2}\left(\mathrm{X}_{1}, \mathrm{X}_{2}\right)} \\
w_{2}=\frac{\mathrm{R}\left(g, \mathrm{X}_{2}\right)-\mathrm{R}\left(\mathrm{X}_{1}, \mathrm{X}_{2}\right) \cdot \mathrm{R}\left(g, \mathrm{X}_{1}\right)}{\mathrm{I}-\mathrm{R}^{2}\left(\mathrm{X}_{1}, \mathrm{X}_{2}\right)}
\end{gathered}
$$

Dans ce cas particulier l'intensité de sélection correspondant à une première troncature sur $\mathrm{X}_{1}$ puis à une troncature sur $\mathrm{X}_{3}$ est obtenue par la formule (Cochran, I95I) (1) :

$$
i_{13}=\frac{\mathrm{R}\left(\mathrm{X}_{1}, \mathrm{X}_{3}\right) y_{1} \mathrm{P}\left(\mathrm{X}>x_{3}^{\prime}\right)+y_{3} \mathrm{P}\left(\mathrm{X}>x_{1}^{\prime}\right)}{\alpha_{1} \alpha_{3}}
$$

où $y_{1}$ et $y_{3}$ désignent les ordonnées de la distribution normale aux points de troncature $x_{1}$ et $x_{3}$ sur les variables $\mathrm{X}_{1}$ et $\mathrm{X}_{3}$ et pour des taux de sélection respectifs de $\alpha_{1}$ et $\alpha_{3}$. $x_{1}$ et $x_{3}$ peuvent

(1) Un programme de calcul automatique de $i_{13}$, connaissant $\alpha_{1} \alpha_{3}, \mathrm{R}\left(\mathrm{X}_{3}\right)$, et $\alpha_{1}$ a été établi (OLLIVIER, MOCQUOT). 


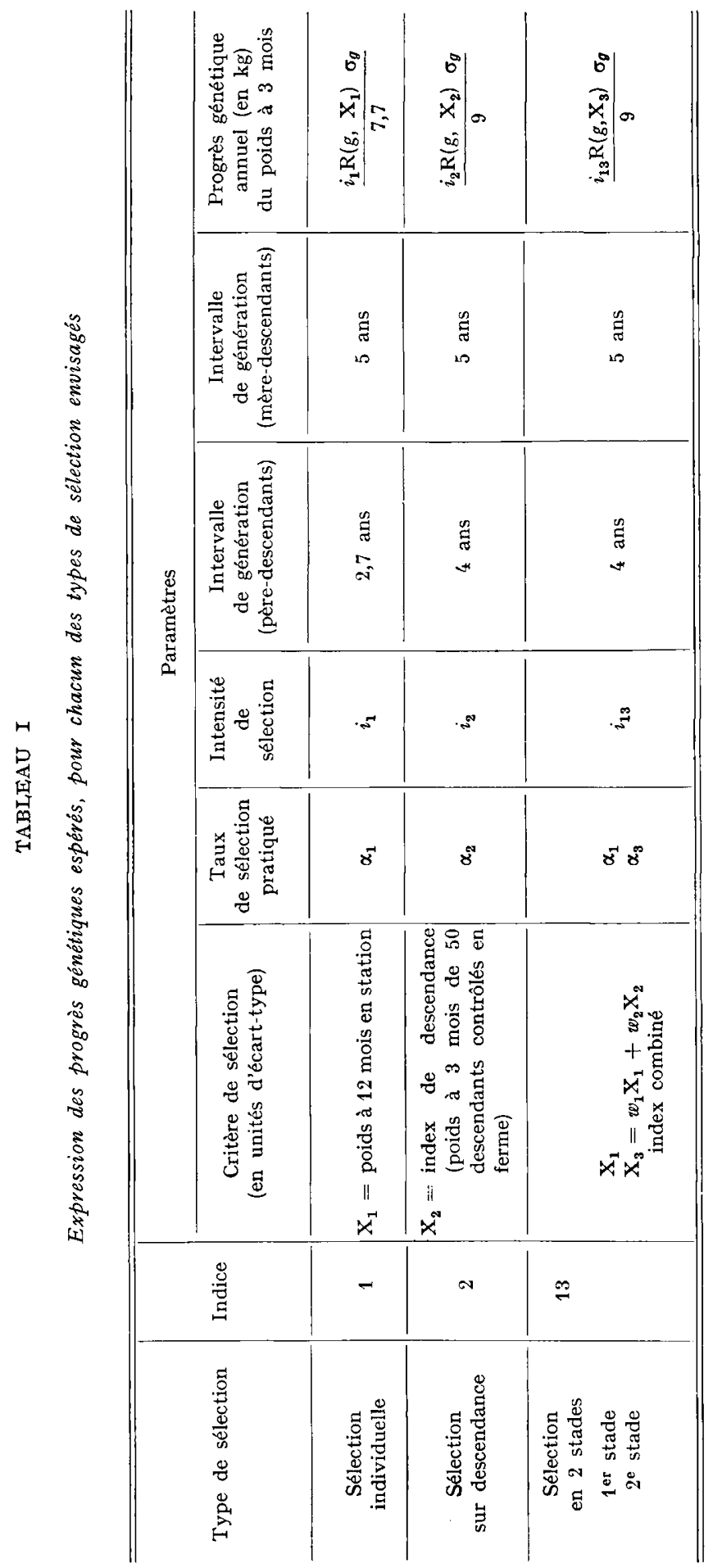


être déterminés pour une loi normale à 2 variables connaissant $R\left(X_{1}, X_{3}\right)$, le taux de sélection $\left(\alpha_{1}\right)$ au premier stade et le taux de sélection total $\left(\alpha_{1} \alpha_{3}\right)$.

$\mathrm{P}\left(\mathrm{X}>x_{i}^{\prime}\right)$ désigne l'intégrale de la loi normale entre $x_{i}^{\prime}$ et l'infini, et :

$$
\begin{aligned}
& x_{3}^{\prime}=\frac{x_{3}-x_{1} \cdot \mathrm{R}\left(\mathrm{X}_{1}, \mathrm{X}_{3}\right)}{\sqrt{\mathrm{I}-\mathrm{R}^{2}\left(\mathrm{X}_{1}, \mathrm{X}_{3}\right)}} \\
& x_{1}^{\prime}=\frac{x_{1}-x_{3} \cdot \mathrm{R}\left(\mathrm{X}_{1}, \mathrm{X}_{3}\right)}{\left.\sqrt{\mathrm{I}-\mathrm{R}^{2}\left(\mathrm{X}_{1}, \mathrm{X}_{3}\right.}\right)}
\end{aligned}
$$

Tous les coefficients de corrélation nécessaires s'expriment simplement en fonction du coefficient de corrélation $\mathrm{R}\left(\mathrm{X}_{1}, \mathrm{X}_{2}\right)$ entre la performance en station à $\mathrm{I} 2$ mois et l'index de descendance du poids à 3 mois, de $\mathrm{R}\left(g, \mathrm{X}_{2}\right)=\sqrt{\frac{0,25 k h_{2}^{2}}{\mathrm{I}+(k-\mathrm{I}) 0,25 h_{2}^{2}}}$ corrélation entre $g$ et cet index de descendance estimé sur le poids à 3 mois de $k$ descendants, $h_{2}^{2}$ représentant l'héritabilité de ce critère :

et

$$
\begin{aligned}
& \mathrm{R}\left(g, \mathrm{X}_{1}\right)=\mathrm{R}\left(\mathrm{X}_{1}, \mathrm{X}_{2}\right) / \mathrm{R}\left(g, \mathrm{X}_{2}\right) \\
& \mathrm{R}\left(g, \mathrm{X}_{3}\right)=\sqrt{\frac{\mathrm{R}^{2}\left(g, \mathrm{X}_{1}\right)+\mathrm{R}^{2}\left(g, \mathrm{X}_{2}\right)-2 \mathrm{R}^{2}\left(\mathrm{X}_{1}, \mathrm{X}_{2}\right)}{\mathrm{r}-\mathrm{R}^{2}\left(\mathrm{X}_{1}, \mathrm{X}_{2}\right)}} \\
& \mathrm{R}\left(\mathrm{X}_{1}, \mathrm{X}_{3}\right)=\mathrm{R}\left(g, \mathrm{X}_{1}\right) / \mathrm{R}\left(g, \mathrm{X}_{3}\right)
\end{aligned}
$$

Connaissant ces valeurs nous sommes en mesure de calculer le progrès génétique pour chacun des types de sélection considérés.

b) Calcul des recettes actualisées.

Les recettes procurées par la sélection proprement dite correspondent à l'accroissement du poids des descendants croisés, donc à la moitié du progrès génétique annuel. Si $\mathrm{H}$ désigne le bénéfice obtenu par unité de production supplémentaire ( $\mathrm{kg}$ vif), si $\mathrm{K}$ désigne le nombre d'inséminations de croisement réalisées chaque année et $V$ le nombre de veaux commercialisés par insémination réalisée, la recette annuelle $\left(\mathrm{R}_{a_{i}}\right)$ procurée par la sélection est de la forme :

$$
\mathrm{R}_{a i}=\mathrm{H} \cdot \mathrm{K} \cdot \mathrm{V} \cdot \Delta \mathrm{G}_{i} / 2
$$

Pour un cycle de sélection donné, visant à fournir l'effectif de reproducteurs nécessaires au renouvellement annuel, la première recette est acquise la llème année et supposée constante jusqu'à la $t$ lème année. Dès lors la recette globale $\mathrm{R}_{i}$ correspondant à un cycle de sélection est égale à la somme des recettes annuelles actualisées sur $t$ années par rapport à l'année initiale du cycle, soit :

$$
\mathrm{R}_{i}=\mathrm{R}_{a i}\left(r^{l-1}+r^{l}+\cdots+r^{t-1}\right)=\mathrm{R}_{a i} \frac{r^{l-1}\left(\mathrm{I}-r^{t-l+1}\right)}{\mathrm{I}-r}
$$

où $r$ désigne le facteur d'actualisation lié au taux d'actualisation $\theta$ par $r=\mathrm{I} /(\mathrm{I}+\theta)$.

Pour tous les systèmes de sélection envisagés dans cette étude, le progrès génétique annuel ne dépend pas du mode de sélection et d'utilisation des taureaux en croisement dès l'instant où ces derniers sont issus du schéma, donc choisis sur ascendance et utilisés de façon constante. De ces valeurs dépend, par contre, le délai entre l'engagement des dépenses et la perception des recettes supplémentaires imputables au progrès génétique annuel; c'est-à-dire le délai entre le moment où l'on commence la sélection des taureaux "pères " utilisés en race pure et celui où sont commercialisés les descendants croisés de leurs fils utilisés, eux, en croisement. Dans le cadre de nos hypothèses un tel délai est réduit aux valeurs minimales de 7 ans dans le cas de sélection sur descendance ou en 2 étapes et de 6 ans dans le cas de sélection individuelle.

\section{C. - Expression des coûts de la sélection}

La nécessité pour le centre de mise en place de loger, d'entretenir et de récolter chaque année le nombre de mâles nécessaires à la réalisation des inséminations de croisement a des répercussions importantes sur la façon de décompter les coûts de la sélection proprement dite.

Dans le cadre des hypothèses faites quant à l'utilisation des mâles en croisement, la durée 
d'utilisation de ces derniers détermine en particulier le nombre de taureaux nécessaires à l'issue du contrôle de spermatogenèse et par voie de conséquence le nombre d'animaux disponibles pour une sélection ultérieure ainsi que le taux maximum de sélection pratiquable à ce stade sur tout critère contrôlé avant I 8 mois.

Nous avons admis, pour chaque méthode de sélection envisagée, que les dépenses à la charge du centre de production étaient celles afférentes à la production de taureaux retenus à l'issue du contrôle de spermatogenèse et à la sélection ultérieure pratiquée parmi ces derniers pour le choix des $n$ taureaux utilisés annuellement en vue d'inséminer le noyau B de femelles. Les coûts de ces différents types de sélection seront légèrement surestimés puisqu'en l'absence de sélection le centre de mise en place, pour fonctionner, devrait lui-même supporter les frais de renouvellement annuel de sa taurellerie, ces derniers étant principalement liés à la nécessité d'éliminer une fraction des taureaux sur la valeur de leur sperme avant leur utilisation intensive. Compte tenu de cette restriction et en admettant que la sélection n'influence pas le volume des inséminations à réaliser, ni le potentiel de production de sperme des taureaux, les coûts de la sélection peuvent être considérés comme indépendants des charges supportées par le centre de mise en place ; ces dernières, constantes pour tous les systèmes de sélection, ne seront pas comptabilisées ici.

Il est difficile de répondre à la fois au besoin d'une modélisation simple et au souci de se conformer à ce que l'on observe au niveau des réalisations concrètes de ces différents types de contrôle et de sélection. Néanmoins chacun des cycles de sélection envisagés peut être décomposé en périodes fixes. A chacune d'elles correspond un coût déterminé par animal compte tenu de la durée de cette période, des installations nécessaires, des contrôles effectués, de la valeur marchande des animaux au début et à la fin de la période considérée et des frais d'entretien de ces animaux pendant cette période.

Nous avons admis, pour les frais correspondants aux installations, que les investissements initiaux (I) par place étaient introduits sous forme d'un amortissement annuel constant $\left(\mathrm{I}_{a}\right)$ sur $t$ années et tel que le cumul, sur les $t$ années, de cet amortissement, actualisé par rapport à l'année initiale, soit égal à la valeur initiale de l'investissement $: \mathrm{I}_{a}=\mathrm{I}(\mathrm{I}-v) /\left(\mathbf{I}-v^{\mathrm{t+1}}\right)$.

Cette hypothèse paraît tout à fait acceptable si les bâtiments nécessaires à la sélection existent déjà. Les frais à considérer, comme liés aux installations pour une période donnée, seront donc proportionnels à cet amortissement $I_{a}$ et inversement proportionnels au taux de rotation annuel des installations correspondant à cette période. Nous avons admis des taux de rotation de 2 et I pour des périodes de durée respectives de 6 mois et $\mathrm{I}$ an. Nous avons admis par ailleurs que pour chacun des trois cycles considérés les animaux étaient achetés à 6 mois d'âge et que leur valeur marchande à un âge supérieur correspondait à la valeur bouchère d'un animal réformé à cet âge.

Si on suppose enfin que tous les frais intervenant au cours d'un cycle donné sont actualisés

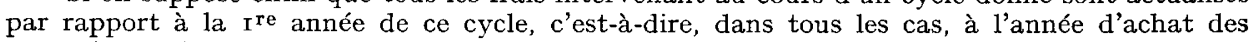
veaux à 6 mois, on peut établir le coût par animal pour différents types de période en supposant l'animal acheté au début de la période, entretenu et contrôlé pendant cette période et vendu à la fin.

\section{Soit les coûts actualisés :}

A pour la période 6-I2 mois avec contrôle individuel en station,

B pour la période I2-I 8 mois avec contrôle de la production de sperme,

C pour la période 6-18 mois sans contrôle individuel mais avec contrôle de la production de sperme,

D pour la période I 8-3o mois, sans testage, l'animal étant à la charge du centre de production pour l'entretien et le logement,

E pour la période $\mathbf{I} 8$ mois- $(\mathbf{1 , 5}+u)$ ans, l'animal étant utilisé $u$ années en croisement par le centre de mise en place donc à sa charge pour le logement, l'entretien et la récolte,

$\mathrm{F}$ coût par animal du contrôle de descendance en ferme sur 50 produits,

$\mathrm{G}$ coût du stockage pendant $\mathbf{I}$ an de la semence d'un taureau récoltée l'année précédente (pendant le testage).

On peut établir les fonctions de coût pour chacune des méthodes de sélection retenues. Compte tenu d'un taux $\mathrm{S}$ de sélection sur la valeur du sperme d'un nombre $n$ de taureaux renouvelés annuellement pour réaliser les accouplements contractuels, d'un nombre permanent $\mathrm{N}$ de taureaux de service utilisés chacun $u$ années en croisement, et des divers taux de sélection définis précédemment on obtient les expressions suivantes :

- Pour un cycle de sélection individuelle :

$$
\mathrm{C}_{1}=\frac{n \mathrm{~A} / \mathrm{S}}{\alpha_{1}}+\frac{\mathrm{N}}{u}(\mathrm{~B} / \mathrm{S}+\mathrm{E})
$$


pour tout

$$
\alpha_{1}<\frac{u n}{N}
$$

—. Pour un cycle de sélection sur descendance :

$$
\mathrm{C}_{21}=n\left(\frac{\mathrm{F}}{\alpha_{2}}+\mathrm{G}\right)+\frac{\mathrm{N}}{u} \quad(\mathrm{C} / \mathrm{S}+\mathrm{E})
$$

pour tout

$$
\alpha_{2}>\frac{u n}{N}
$$

et

pour tout

$$
\mathrm{C}_{22}=n\left(\frac{\mathrm{C} / \mathrm{S}+\mathrm{F}+\mathrm{D}}{\alpha_{2}}+\mathrm{G}\right)+\frac{\mathrm{N}}{u}(\mathrm{E}-\mathrm{D})
$$

$$
\alpha_{2}<\frac{u n}{N}
$$

et enfin pour un cycle de sélection en 2 stades :

si

$$
\mathrm{C}_{31}=n\left(\frac{\mathrm{A} / \mathrm{S}}{\alpha_{1} \alpha_{3}}+\frac{\mathrm{F}}{\alpha_{3}}+\mathrm{G}\right)+\frac{\mathrm{N}}{u}(\mathrm{~B} / \mathrm{S}+\mathrm{E})
$$

et

$$
\alpha_{3}>\frac{u n}{\mathrm{~N}}
$$

si

$$
\mathrm{C}_{32}=n\left(\frac{\mathrm{A} / \mathrm{S}}{\alpha_{1} \alpha_{3}}+\frac{\mathrm{B} / \mathrm{S}+\mathrm{D}+\mathrm{F}}{\alpha_{3}}+\mathrm{G}\right)+\frac{\mathrm{N}}{u}(\mathrm{E}-\mathrm{D})
$$

$$
\alpha_{3}<\frac{u n}{\mathrm{~N}}
$$

Les situations pour lesquelles les expressions $\mathrm{C}_{22}$ et $\mathrm{C}_{32}$ sont applicables correspondent en effet à la mise en testage d'un certain nombre de mâles non utilisés en croisement et éliminés par la suite, donc à des frais supplémentaires pour le centre de production.

\section{D. - Conventions de travail}

L'expression des gains et des frais fait apparaître un nombre de facteurs trop grand pour permettre une recherche analytique précise et simple des solutions optimales, aussi les paramètres ont-ils été divisés en 3 catégories, suivant leur variabilité possible, leur maîtrise éventuelle par le centre de production et enfin la précision avec laquelle ils sont connus a priori.

Le ou les taux de sélection, étant presque totalement contrôlables par le centre de production, ont été considérés comme des variables continues susceptibles de prendre dans chaque cas n'importe quelle valeur de leur domaine possible.

La durée d'utilisation et les conditions d'actualisation peuvent, en partie, varier par suite d'options prises par le centre de production. Les paramètres génétiques sont estimés avec une précision relatıvement faible et risquent d'être différents selon la population sélectionnée. Pour aboutir à une sulution généralisable, il était important d'estimer l'influence de ces facteurs sur les résultats techniques et économiques. Pour cela nous avons successivement attribué à chacun d'entre eux un nombre fixe de valeurs discontinues, les autres paramètres restant constants.

Enfin une valeur fixe a été attribuée au reste des paramètres correspondant à un centre de production produisant annuellement $K=450$ ooo doses de sperme dont l'utilisation en croisement conduit à la commercialisation de 250000 veaux de boucherie chaque année. $(\mathrm{V}=0,555$ veau par insémination première.) En supposant un volume annuel de I 5 ooo doses récoltées et utilisées par taureau sélectionné, $\mathrm{N}=30$ taureaux doivent être récoltés en permanence. Dans le but de limiter l'accroissement du taux de consanguinité nous avons admis que dans tous les cas $n=5$ taureaux seraient sélectionnés annuellement pour réaliser les accouplements contractuels avec le noyau $\mathrm{B}$ de femelles. Ceci correspond à un accroissement de 0,33 et 0,18 p. Ioo par an du taux de consanguinité respectivement en sélection individuelle et sur descendance (ou en 2 étapes). Le taux d'élimination sur la production de sperme (I - S) peut être relativement variable selon les races et types génétiques une valeur fixe de $\mathrm{r} / 3$ a été choisie. 
Pour le calcul des recettes nous avons admis un bénéfice net $(\mathrm{H})$ de 5 francs par $\mathrm{kg}$ vif supplémentaire du poids à 3 mois et une variance de ce poids de 4 oo. Pour le décompte des coûts d'entretien et de contrôle des animaux nous avons obtenu les valeurs non actualisées suivantes : $\mathrm{A}=2770, \mathrm{~B}=2220, \mathrm{C}=4660, \mathrm{D}=3900, \mathrm{E}=-\mathrm{I}$ 100, $\mathrm{F}=8500, \mathrm{G}=75^{\circ}$ francs, en supposant les bâtiments amortis sur 20 ans à $\mathbf{I} 5 \mathrm{p}$. roo et $u=\mathrm{I}$ an.

\section{E. - Critères de choix entre les différentes méthodes de sélection}

Le critère économique retenu est le bénéfice actualisé (gains - frais). Plutôt que de l'exprimer directement en fonction des variables de sélection (taux de sélection), nous avons préféré le donner en fonction du coût de production. En effet, le montant des dépenses engagées constitue un facteur toujours important et souvent limitant pour le centre de production.

Dans le cas de sélection individuelle ou sur descendance, il existe une relation bi-univoque entre coûts, bénéfices et taux de sélection pour une valeur fixée des autres paramètres. Dans la sélection en 2 étapes, à un même coût peuvent correspondre différents bénéfices, suivant les combinaisons entre les taux de sélection à chacune des 2 étapes, même pour une valeur fixée des autres paramètres. Dans ce cas nous avons calculé le couple de valeurs des deux taux rendant le bénéfice maximum pour une dépense fixée.

De cette manière, à une dépense donnée correspond toujours le bénéfice maximum permis pour chaque situation envisagée, ce qui permet de la comparer directement aux autres cas étudiés.

Pour une plus grande généralité des résultats, les coûts et les bénéfices ont été exprimés en francs par insémination première.

\section{IV. - RÉSULTATS ET DISCUSSIONS}

Sauf indication contraire, les courbes du bénéfice maximum en fonction du coût ont été établies en adoptant les valeurs les plus vraisemblables pour les paramètres $\left(\theta=\mathrm{I} 5 \mathrm{p}\right.$. IOO, $t=20$ ans, $u=\mathrm{I}$ an, $h_{2}^{2}=0$,I0, GAIL $\mathrm{I}_{1} \mathrm{ARD}, \mathrm{I} 97 \mathrm{I}, \mathrm{R}\left(\mathrm{X}_{1}, \mathrm{X}_{2}\right)=0,3$ Poujardieu et VISSAC, I968). Nous avons étudié successivement l'influence du taux d'actualisation, de la durée d'actualisation, de la durée d'utilisation des taureaux en croisement, et enfin des valeurs de l'héritabilité et de la corrélation.

Pour chaque situation nous avons reporté sur les figures les taux de sélection autour du maximum (taux global pour la sélection combinée).

\section{A. - Influence du taux d'actualisation}

Le choix du taux d'actualisation est une option prise par l'entrepreneur décidé à investir. Il permet de comparer la rentabilité de l'investissement considéré à celle d'un placement bancaire au même taux. Le choix d'un taux élevé favorise les recettes immédiates par rapport à celles obtenues plus tardivement et permet ainsi, dans une certaine mesure, de tenir compte des incertitudes économiques concernant l'avenir et des risques afférents à l'opération envisagée. Dans le cadre de notre étude, compte tenu des risques encourus lors d'un investissement dans un matériel " biologique ", fondamentalement sujet à des influences aléatoires d'une part, et des incertitudes du marché du veau de boucherie pour l'avenir d'autre part, telle méthode de sélection ne pourra être considérée comme rentable que si elle procure des bénéfices pour un taux élevé d'actualisation.

L'influence $\mathrm{du} \operatorname{taux}(\theta=0 \mathrm{p}$. Ioo, $\theta=8 \mathrm{p}$. Ioo et $\theta=$ I5 p. Ioo) sur la rentabilité apparaît sur la figure 2. Par rapport à un taux nul, le bénéfice diminue pratiquement des trois quarts pour un taux de $8 \mathrm{p}$. Ioo et des sept huitièmes pour 
un taux de I5 p. Ioo. A l'optimum, le pourcentage de mâles à conserver est une fonction du taux d'actualisation, il augmente pratiquement dans un rapport de $\mathrm{I}$ à 2 ou 3 pour les valeurs $0 ; 8$ et I5 p. Ioo de $\theta$.

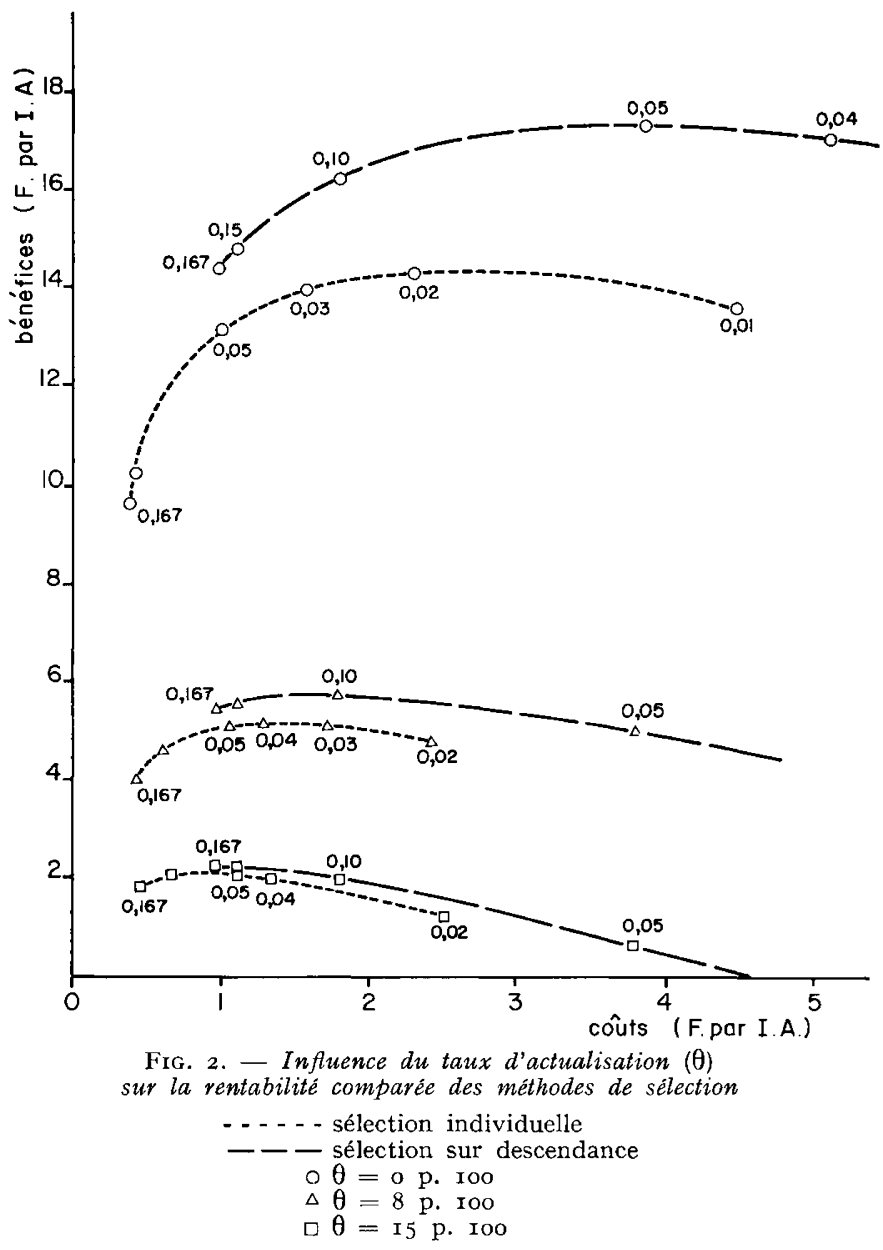

Dans tous les cas, le contrôle sur descendance est supérieur au contrôle individuel, bien que la différence soit faible en particulier pour des taux élevés et/ou un niveau de dépenses faible. Si ce dernier est très bas seul le contrôle individuel est envisageable. A l'optimum, on remarque qu'il faut conserver un pourcentage de mâles dans le cas du contrôle de descendance à peu près trois fois supérieur au taux de sélection individuelle.

\section{B. - Influence de la durée d'actualisation}

La figure 3 indique que de Io ans (période minimum) à 20 ans (durée couramment admise en sélection), le bénéfice est à peu près triplé - l'augmentation est beaucoup moins sensible au-delà de 20 ans. Le taux optimum de sélection reste 
stable pour le contrôle de descendance et diminue en raison inverse de la durée d'actualisation pour le contrôle individuel.

Il est intéressant de remarquer que le classement des méthodes de choix des reproducteurs est inversé pour une période d'environ 15 ans : le contrôle individuel étant supérieur en deçà et inférieur au-delà. Cependant les différences entre systèmes restent toujours faibles.

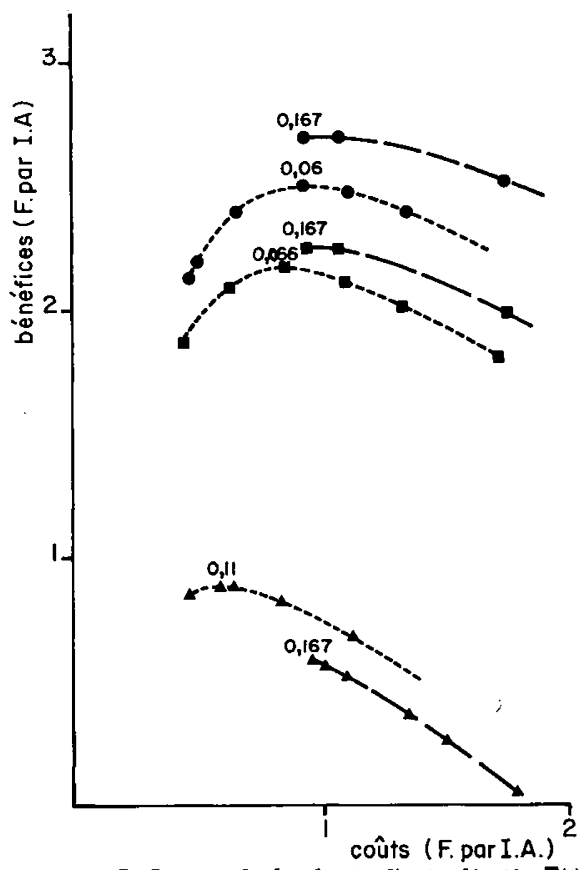

FIG. 3. - Influence de la durée d'actualisation $\mathbf{I}(t)$ sur la rentabilité comparée des méthodes de sélection

$$
\begin{aligned}
& -- \text { sélection individuelie } \\
& \text { o } t \text { infinie } \\
& \square t=20 \text { ans } \\
& \Delta t=10 \text { ans }
\end{aligned}
$$

En comparant les résultats obtenus pour les variations des conditions d'actualisation, on peut admettre qu'elles modifient largement le bénéfice escompté mais finalement assez peu le rapport des bénéfices attendus pour chacune des méthodes de sélection étudiées. Aussi avons-nous retenu les conditions de sécurité maximum pour ce type d'investissement, à savoir $15 \mathrm{p}$. Ioo sur 20 ans.

\section{C. - Infuence de la durée minimale d'utilisation en croisement}

Les courbes de bénéfice sont reportées sur la figure 4, pour une sélection individuelle, sur descendance et en deux étapes des mâles utilisés en race pure avec une durée d'utilisation de $\mathrm{I}$ et 4 ans des taureaux non sélectionnés destinés au croisement.

Dans cette hypothèse, les différences de bénéfice et d'intensité de sélection n'apparaissent pas très importantes, la meilleure durée d'utilisation variant avec 
le type de sélection. En contrôle individuel, l'allongement de la période est favorable, car si le nombre de taureaux entrant en station est à peu près identique, la sélection à la sortie de station est plus forte ce qui réduit les frais pour la période de contrôle de la production de semence. Pour le contrôle de descendance, et pour un même

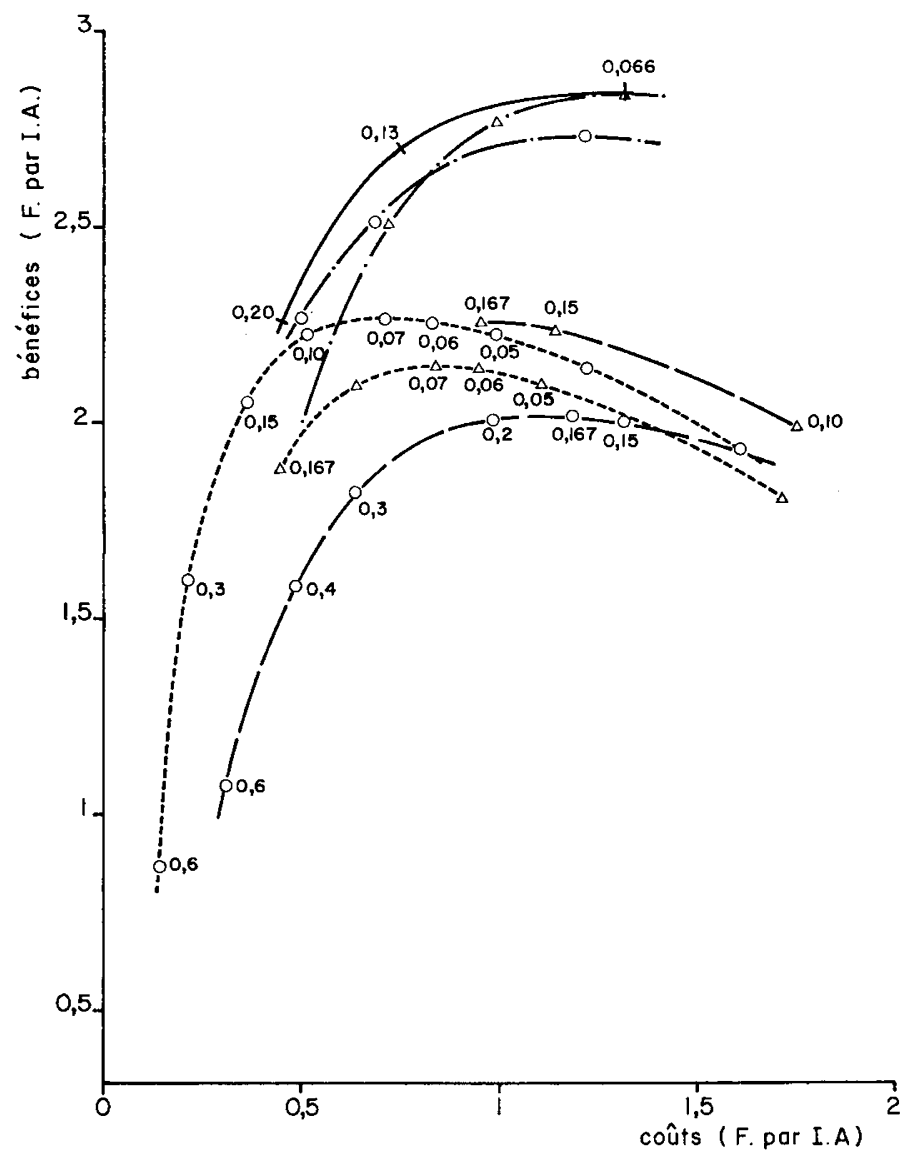

FIG. 4. - Influence de la durée minimale d'utilisation des mâles en croisement (u) sur la rentabilité des différentes méthodes de sélection des mâles utilisés en race pure

$$
\begin{aligned}
& -1-1-\text { sélection individuelle } \\
& \Delta u=1 \text { an } \\
& -1-\text { sélection sur descendance } 2 \text { étapes }
\end{aligned}
$$

nombre de mâles mis à l'épreuve, si la durée d'utilisation augmente, les frais augmentent également puisque le centre est conduit à entretenir et récolter un plus grand nombre de taureaux chaque année, pour ne mettre en place qu'une fraction de leur semence. Dans ce cas une utilisation prolongée est économiquement défavorable, tout au moins sous l'hypothèse d'un choix au hasard des taureaux retenus pour le croisement parmi ceux mis à l'épreuve.

La sélection en 2 étapes faisant intervenir les deux tendances opposées précé- 
dentes, il existe un point d'intersection pour les courbes représentatives des deux durées considérées. Dans ce cas, en dessous d'un investissement minimum (de l'ordre de $0,80 \mathrm{~F} / \mathrm{IA}$ ), une utilisation prolongée est préférable. La comparaison deux à deux de đurées d'utilisation différentes conduit aux mêmes constatations. Globalement, pour la sélection en deux étapes, il existe une courbe optimale des bénéfices telle que l'augmentation des coûts s'accompagne d'une réduction de la durée minimale d'utilisation des taureaux en croisement et d'une augmentation des bénéfices jusqu'à l'optimum. Ce dernier est atteint pour des coûts voisins de I,30 F/IA et une durée d'utilisation de $I$ an. Les bénéfices augmentent rapidement pour des coûts inférieurs à $0,75 \mathrm{~F} / \mathrm{IA}$ et des durées inférieures à 2 ans et lentement entre cette même valeur et 1'optimum pour décroître faiblement au-delà.

Si l'on se réfère à l'incidence de ce facteur sur les intensités de sélection optimales on constate que seul le taux de sélection au deuxième stade varie avec la durée d'utilisation des mâles pour le croisement, donc en fonction des coûts. En effet dans le cadre de nos hypothèses, selon lesquelles la variation de la durée d'utilisation ne modifie pas les recettes escomptées, le bénéfice obtenu pour une intensité de sélection globale donnée sera maximum si les coûts sont minimum, donc si toute la semence des taureaux soumis au testage est mise en place en croisement. On constate ainsi une correspondance exacte entre la durée optimale d'utilisation et le taux de sélection au $2^{\mathrm{e}}$ stade pour un nombre fixe de taureaux retenus à l'issue du contrôle de descendance.

\section{D. - Influence de la variation des paramètres génétiques}

Nous avons reproduit sur la figure 5 les courbes des bénéfices maximum en fonction des coûts pour les 3 types de sélection et pour certaines combinaisons des valeurs des coefficients d'héritabilité $\left(h_{2}^{2}\right)$ et de corrélation $\left(\mathrm{R}\left(\mathrm{X}_{1}, \mathrm{X}_{2}\right)\right)$. Nous avons considéré ces deux paramètres comme indépendants, car le coefficient $\mathrm{R}$ dépend à la fois des héritabilités, à 3 mois en ferme, à 12 mois en station, et du coefficient de corrélation génétique entre ces deux poids.

Dans tous les cas les frais de sélection sont d'autant mieux valorisés que l'héritabilité est plus forte. La pression de sélection optimale et les coûts, qui lui sont liés, varient également dans le même sens. Ces différences sont, cependant, un peu moins marquées pour le contrôle individuel. Le mérite relatif des méthodes proposées n'est pas le même suivant les combinaisons des deux paramètres étudiées. La sélection en deux étapes est toujours meilleure que le choix sur descendance, elle est presque toujours préférable à un choix individuel sauf pour un coefficient d'héri-

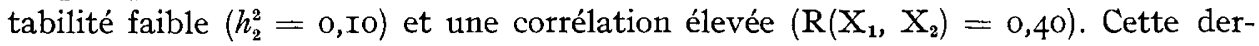
nière situation est peu probable, car elle suppose une héritabilité en station forte et une corrélation génétique entre poids à 3 mois en ferme et poids à 12 mois en station élevée.

Le choix individuel est nettement inférieur au choix sur descendance si la corrélation $\mathrm{R}\left(\mathrm{X}_{1}, \mathrm{X}_{2}\right)$ est faible et/ou l'héritabilité $h_{2}^{2}$ élevée. Il lui est sensiblement égal pour les deux couples de valeurs : $h_{2}^{\prime}=0$, I0 $; \mathrm{R}\left(\mathrm{X}_{1}, \mathrm{X}_{2}\right)=0,3$ ou $h_{2}^{2}=0,20$; $\mathrm{R}\left(\mathrm{X}_{1}, \mathrm{X}_{2}\right)=0,4$. Il faut cependant noter à nouveau que pour des disponibilités financières très faibles, seules les méthodes faisant intervenir un contrôle de performance individuelle sont possibles. 


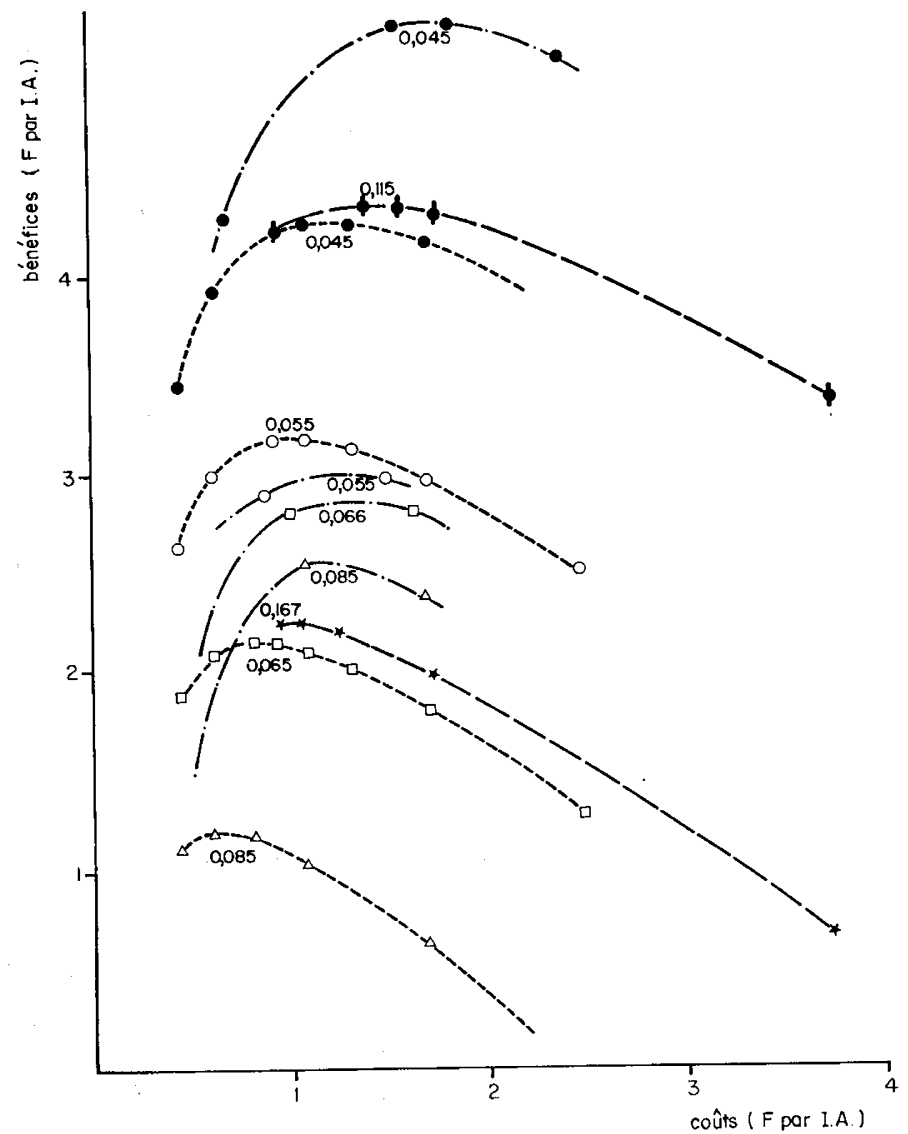

Frg. 5. - Influence des paramètres génétiques sur la rentabilité comparée des méthodes de sélection

\begin{tabular}{|c|c|c|c|c|}
\hline Héritabilité du poids à 3 mois & \multicolumn{3}{|c|}{0,10} & 0,20 \\
\hline - - - - sélection sur descendance & \multicolumn{3}{|c|}{$\star$} & i \\
\hline - sélection individuelle & $\Delta$ & $\square$ & 0 & $\bullet$ \\
\hline $\begin{array}{l}\text { Corrélation entre le poids à } 12 \text { mois } \\
\text { et l'index descendance du poids à } \\
3 \text { mois }\end{array}$ & 0,2 & 0,3 & 0,4 & 0,4 \\
\hline
\end{tabular}

Les chiffres portés sur le graphique indiquent le taux global de sélection au voisinage de l'optimum. 
Si on se situe près de l'optimum pour une valeur constante de l'héritabilité, on constate sur la figure 5 que les variations du bénéfice en fonction de la corrélation $\mathrm{R}\left(\mathrm{X}_{1}, \mathrm{X}_{2}\right)$ sont relativement faibles pour la sélection combinée au contraire de la sélection individuelle. La même tendance ne peut être relevée à propos des taux de séleçtion. Le tableau 2 indique en effet une similitude notable des taux globaux de sélection pratiqués lors d'une sélection individuelle et en deux étapes pour une même combinaison des paramètres génétiques. Par ailleurs, on note une stabilité remarquable du taux de sélection sur index combiné au $2^{\mathrm{e}}$ stade dans le cas de sélection en deux étapes, du moins tant que les deux paramètres considérés ne présentent pas les valeurs extrêmes et opposées pour lesquelles nous avons constaté une supériorité du contrôle individuel sur le contrôle combiné. La valeur de I/6 observée à 1'optimum pour le taux de sélection au $2^{\mathrm{e}}$ stade est liée aux hypothèses que nous avons faites quant au nombre de taureaux conservés, et représente à la fois un optimum technique et un optimum économique pour les combinaisons les plus probables des paramètres génétiques étudiés.

TABLEAU 2

Taux de sélection optimum selon le mode de sélection envisagé et la valeur des paramètres génétiques

\begin{tabular}{|c|c|c|c|c|c|}
\hline & Taux & \multicolumn{4}{|c|}{$h_{2}^{2}$} \\
\hline & de sélection & \multicolumn{3}{|c|}{0,10} & 0,20 \\
\hline $\begin{array}{l}\text { Sélection } \\
\text { sur descendance }\end{array}$ & $\alpha_{2}$ & \multicolumn{3}{|c|}{0,167} & 0,115 \\
\hline $\begin{array}{l}\text { Sélection } \\
\text { individuelle }\end{array}$ & $\alpha_{1}$ & 0,085 & 0,065 & 0,055 & 0,045 \\
\hline \multirow[t]{2}{*}{$\begin{array}{l}\text { Sélection } \\
\text { en deux étapes }\end{array}$} & $\begin{array}{c}\alpha_{1} \\
\alpha_{3} \\
\alpha_{1} \alpha_{3}\end{array}$ & $\begin{array}{l}0,5 \\
0,167 \\
0,085\end{array}$ & $\begin{array}{l}0,4 \\
0,167 \\
0,065\end{array}$ & $\begin{array}{l}0,24 \\
0,23 \\
0,055\end{array}$ & $\begin{array}{l}0,25 \\
0,167 \\
0,045\end{array}$ \\
\hline & $R\left(X_{1} X_{2}\right)$ & 0,2 & 0,3 & 0,4 & $0,,^{\prime}$ \\
\hline
\end{tabular}

\section{DISCUSSION GÉNÉRALE E'T CONCLUSION}

Nous avons volontairement, restreint le champ de notre étude aux répercussions à long terme de la sélection. En effet, nos hypothèses principales reviennent à admettre que les mâles utilisés pour la production commerciale de veaux n'ont fait l'objet d'aucune sélection efficace pour le critère considéré en dehors du fait qu'ils sont issus de vaches du noyau de sélection accouplées à des taureaux choisis d'après l'une des méthodes proposées. En d'autres termes, nous avons seulement considéré une sélection des ascendants mâles. Cette restriction est évidemment 
importante; nous montrons par exemple (annexe) que la rentabilité est notablement augmentée lorsque leurs résultats en station ou ceux de leur descendance servent à choisir les mâles non seulement pour le noyau de race pure mais également pour la production commerciale de veaux sur le reste du cheptel.

Malgré cela notre étude met en évidence l'intérêt d'une sélection sur les ascendants, susceptible d'entraîner à elle seule une progression de I p. Ioo environ par an du niveau génétique moyen des mâles achetés par le centre de production de semence, avec un taux de rentabilité des capitaux investis de l'ordre de $200 \mathrm{p}$. Ioo. Parmi les méthodes étudiées, la sélection combinée en deux étapes nous semble la meilleure. D’abord parce qu'elle procure les bénéfices les plus grands, sauf dans le cas, assez peu probable, de coefficients d'héritabilité ayant une valeur basse en ferme et élevée en station et d'une corrélation génétique forte entre le poids à 3 mois en ferme et celui à I2 mois en station. Ensuite parce qu'autour de l'optimum, les bénéfices et les intensités de sélection sont moins sensibles à des variations des paramètres génétiques que dans le cas des autres méthodes. Enfin, parce qu'elle laisse au centre de production de semence une certaine souplesse dans l'importance relative des contrôles individuels ou sur descendance. Globablement, ces deux derniers types de contrôle, envisagés séparément, semblent avoir des rentabilités équivalentes, à long terme du moins.

Si le contrôle de descendance paraît préférable à la sélection individuelle pour les valeurs les plus probables des paramètres étudiés, celle-ci présente un avantage certain lorsque les disponibilités financières sont faibles - cas des races de faible effectif —, le coût de l'argent élevé, ou l'échéance de rentabilité brève — cas d'une spéculation d'avenir incertain.

Notre étude met ainsi en évidence la grande importance des conditions économiques (taux et durée d'actualisation, bénéfices et cô̂ts unitaires) sur la rentabilité des opérations de sélection. Nous avons, en particulier, montré que les recettes espérées au-delà d'une période dépassant 20 ans, n'interviennent pratiquement plus d'un point de vue économique. Ce délai est, en fait, bref pour un généticien puisqu'il représente seulement 4 ou 5 générations chez les bovins.

Dans une étude ultérieure, nous nous proposons d'analyser plus en détail que nous ne l'avons fait dans l'annexe I, les répercussions d'un choix direct des mâles utilisés pour la production de veaux. Il est en effet probable, compte tenu des remarques précédentes, que dans ce cas le plan de sélection optimal ne sera plus identique à celui que nous avons proposé ici, puisque les recettes à court terme interviendront relativement plus que celles à long terme du fait de 1'actualisation. Nous insistons cependant encore sur l'intérêt de la sélection sur ascendance telle que nous l'avons décrite, tant du point de vue génétique pour l'amélioration de notre cheptel commercial, que du point de vue économique, même si on ne veut tenir compte que de ce qui se passe à une échéance relativement proche, d'environ I5 ans et dans des conditions assez défavorables — coût de l'argent élevé.

A notre avis, des raisonnements analogues à ceux que nous avons tenus ici, seraient d'une grande utilité pour rationaliser les choix budgétaires qui doivent être faits à propos des programmes de sélection relatifs à d'autres espèces que les bovins, ou pour ces derniers à des spéculations différentes, telles que la production de jeunes bovins ou la production laitière. 


\title{
REMERCIEMENTS
}

Nous remercions M. E. P. Cunningham et M. Poutous pour la lecture critique de notre manuscrit.

\author{
SUMMARY \\ INVESTIGATIONS ON THE RENTABILITY OF A SELECTION PLAN \\ FOR A CATTLE STRAIN TO BE USED IN FIRST-GENERATION \\ CROSSING FOR MEAT CALF PRODUCTION
}

A genetico-economic method of reasoning for selection is formulated. Selection of paternal ascendance of bulls is particularly studied ; namely, long term rentability, comparing 3 methods of sire selection at the level of an elementary selection cycle :

- selection on a station performance test (weight at 12 months) ;

- selection on a field progeny test (weight at 3 months) ;

- selection in two stages by successive truncation on weight at $\mathrm{I} 2$ months in the station then on a combined index of the 2 proceeding criteria.

In each case the monetary income distribution in time is taken into account using the discounting procedure, and the conditions for obtaining discounted maximum profit are determined. Then the sensitivity of obtained values to variations of some economic or genetic parameters is analyzed. The results point to the following conclusions :

$I^{0}$ in all cases, selection of male ascendants alone justifies the investment made;

$2^{0}$ the introduction of economic considerations into the genetic study of selection plans permits the methods proposed to be more objectively compared and more realistic optima to be determined for each one ;

$3^{\circ}$ two-stage selection seems to be the best of the methods studied, first, because the largest profits are obtained of a given cost and secondly, because near the optimum, the response is less sensitive to possible variation of the parameters which are badly known or fixed a priori;

$4^{\circ}$ direct selection of bulls used in crossing permits a better rentability of expensives already engaged for ascendance selection.

\section{RÉFÉRENCES BIBLIOGRAPHIQUES}

Cochran W. G., I95I. Improvement by means of selection. Proceedings of the second Berkeley Symposium on Mathematical Statistics and Probability, 449-470.

Frebling J., Gaillard J., Vissac B., I97r. Dix ans de choix et d'utilisation par insémination artificielle de taureaux de races à viande pour la production de veaux de boucherie. Journ. Féd. eur. Zootech. Com. Prod. bov., Versailles, ronéoté.

Gaillard J., I97r. (Communication personnelle).

Hill W. G., I97I. Investment appraisal for national programmes. Anim. Prod., 13, 37-50.

Hinks C. J. M., I970 a. The selection of dairy bulls for AI. Anim. Prod., 12, 569-576.

Hinks C. J. M., I970 $b$. Performance test procedures for meat production amongst dairy bulls used in AI. Anim. Prod., 12, 577-583.

Hinks C. J. M., I97I. The genetic and financial consequences of selection amongst dairy bulls in artificial insemination. Anim. Prod., 13, 209-218.

Lindhe B., I968. Model simulation of AI breeding within a dual purpose breed of cattle. Acta Agr. Scand., 18, 33-4r.

McClintock A. E., Cunningham E. P., I97x. AI breeding strategy for dual purpose cattle populations. Ann. Génét. Sél. anim., 4, г37-138. 
Poujakdieu B., Vissac B., r968. Étude biométrique de la croissance et de la valeur bouchère de veaux croisés charolais et limousin. I. Paramètres génétiques et phénotypiques. Ann. Zootech., 17, I43-I 58 .

Poutous M., VIssac B., I962. Recherche théorique des conditions de rentabilité maximum de l'épreuve de descendance des taureaux d'insémination artificielle. Ann. Zootech., 11, 233-256.

Rendel J. M., Robertson A., I950. Estimation of genetic gain in milk yield by selection in a closed herd of dairy cattle. J. Genet., $50, \mathrm{x}-8$.

Robertson A., I957. Optimum group size in progeny testing and family selection. Biometrics, 4, $442-450$.

Soller M., Bar-Anan R., Pasternak H., r966. Selection of dairy cattle for growth rate and milk production. Anim. Prod., 8, Iog-II9.

Vissac B., Frebling J., Ménissier F., Gaillard J., I97x. Le choix des reproducteurs pour la production de jeunes bovins. Revue Élev. (5), 9 I-Ior. $(6,7), 69-77$.

\section{ANNEXE}

Nous pouvons sous des hypothèses volontairement restrictives, montrer l'intérêt d'utiliser des mâles choisis sur performance individuelle en station ou même testés sur descendance, non seulement sur le noyau de femelles de race pure mais aussi sur les femelles support du croisement.

Comparons, pour la situation de rentabilité optimale de la sélection sur ascendance, deux types de choix et d'utilisation des taureaux destinés au croisement :

I ${ }^{0}$ Supposons que ces derniers soient non plus choisis au hasard mais choisis sur performance individuelle en station et utilisés de 18 à 30 mois.

$2^{\circ}$ Admettons qu'après un même choix individuel, aucun de ces taureaux ne soit utilisé l'année suivant leur sortie de station, mais que la semence soit récoltée et stockée. Admettons également qu'après connaissance des résultats de testage, la moitié d'entre eux soit conservée une année supplémentaire et que les inséminations pour le croisement soient alors faites avec leur semence.

Dans le premier cas, et par rapport à la situation envisagée au cours de la présente étude, les coûts ne sont pas accrus mais il faut prendre en considération les recettes supplémentaires obtenues en croisement de façon ponctuelle la $3^{\mathrm{e}}$ année du cycle de sélection. Ces dernières sont liées à la supériorité génétique des mâles utilisés et ont pour expression :

$$
\mathrm{R}_{\mathrm{S}_{1}}=\frac{\mathrm{K} \cdot \mathrm{V} \cdot \mathrm{H} \cdot \sigma_{g}}{2} \cdot r^{2} i_{1} \mathrm{R}\left(g, \mathrm{X}_{1}\right)
$$

Dans le second cas, et par rapport à l'utilisation immédiate à leur sortie de station, la date de réalisation des recettes, aussi bien ponctuelles que de celles liées au progrès génétique à long terme, est augimentée d'un an. La valeur génétique des mâles utilisés est, par contre, augmentée du fait qu'ils sont choisis sur descendance. Avec actualisation 1'expression des recettes supplémentaires est la suivante :

$$
\mathrm{R}_{\mathrm{S}_{2}}=\frac{\mathrm{K} \cdot \mathrm{V} \cdot \mathrm{H} \cdot \sigma_{g}}{2} \cdot r^{3} \cdot\left(i_{13}^{\prime}-r^{3} \frac{i_{13}}{9}\right) \cdot \mathrm{R}\left(g, \mathrm{X}_{3}\right)
$$

où $i_{13}^{\prime}$ désigne l'intensité de sélection correspondant à une sélection en deux étapes avec des taux respectifs de $\alpha_{1}=0,4$ et $\alpha_{3}=0,5$. 
Pour ce qui concerne les coûts ils sont augmentés du fait que la moitié des taureaux sont conservés et récoltés pendant une année supplémentaire. L'expression des coûts supplémentaires s'écrit :

$$
\mathrm{C}_{\mathrm{S}_{2}}=\frac{\mathrm{N}}{2} r(\mathrm{VR}(\mathrm{I}-r)+r(\mathrm{~F}+\mathrm{G}))
$$

où VR désigne la valeur bouchère d'un mâle de 30 mois,

F désigne les frais de logement, d'entretien et de récolte d'un mâle pendant I an,

G les frais de stockage pendant $\mathrm{I}$ an de la semence, d'un mâle récoltée l'année précédente,

N l'effectif de taureaux retenus à l'issue du contrôle individuel.

Avec les valeurs que nous avons admises précédemment, l'expression par insémination des recettes, des frais, et des bénéfices supplémentaires devient :

$$
\begin{aligned}
& \mathrm{R}_{\mathrm{s}_{1}}=2,566 \mathrm{~F}=\mathrm{B}_{\mathrm{s}_{1}} \\
& \mathrm{R}_{\mathrm{s}_{2}}=4,965 \mathrm{~F} ; \mathrm{C}_{\mathrm{s}_{2}}=0, \mathrm{I} 6 \text { et } \mathrm{B}_{\mathrm{S}_{2}}=4,8
\end{aligned}
$$

et celle des recettes supplémentaires correspondant à un supplément de dépenses :

$$
\mathrm{R}_{\mathrm{s}_{2}}-\mathrm{R}_{\mathrm{S}_{1}}=2,4 \mathrm{~F}
$$

Ces résultats montrent l'intérêt de choisir aussi sur descendance les mâles destinés au croisement, du fait de l'accroissement immédiat mais ponctuel des recettes procuré par cette sélection supplémentaire. Nous devons cependant faire remarquer que cet avantage n'apparaît aussi grand que parce que nous avons attribué au programme de sélection en race pure à long terme l'essentiel des frais. Néanmoins cet aspect mérite d'être approfondi et nous pensons reprendre ce travail avec des hypothèses moins restrictives et mieux adaptées aux exigences de la pratique. 